\title{
Research on Digital Agricultural Information Resources Sharing Plan Based on Cloud Computing*
}

\author{
Guifen Chen ${ }^{1, * *}, \mathrm{Xu} \mathrm{Wang}^{2}$, Hang Chen ${ }^{1}$, Chunan $\mathrm{Li}^{1}$, \\ Guangwei Zeng ${ }^{1}$, Yan Wang ${ }^{1}$, and Peixun Liu ${ }^{1}$ \\ ${ }^{1}$ College of Information and Technology Scince, \\ Jilin Agricultural University Chang Chun, 130118 \\ ${ }^{2}$ College of Computer Science and Technology, \\ Jilin University Chang Chun, 130012 \\ guifchen@163. com
}

\begin{abstract}
In order to provide the agricultural works with customized, visual, multi-perspective and multi-level active service, we conduct a research of digital agricultural information resources sharing plan based on cloud computing to integrate and publish the digital agricultural information resources efficiently and timely. Based on cloud computing and virtualization technology, we establish a cloud computing server storage architecture, design deployment of server virtualization service, and present information resource sharing plan, so the users on different network environment could access to digital agricultural information resources at any time. Application examples demonstrate the use of cloud computing technology can provide heterogeneous resource sharing services for all agriculture-related facilities.
\end{abstract}

Keywords: cloud computing, digital agricultural, information sharing, virtualization.

\section{Introduction}

With the development of agricultural information, agricultural information websites have sprung up. But because of uneven geographical distribution, imperfect management system and other reasons, many sites did not play a real role[1].Digital agriculture as the representative of precision agriculture is an emerging discipline, the domestic service-oriented sites focusing on digital agricultural information and technology are very few and did not use advanced technologies, such as cloud computing, grids and others. They cannot become a professional, efficient, real-information services sites.

Cloud computing is presented in a commercial implementation of grid computing[2]. It is a low cost for providing vast amounts of data processing for many customers, and has important scientific value and commercial value in agricultural

\footnotetext{
Foundation project: National"863"High-tech Project(2006AA10A309), National Spark Project(2008GA661003) and Changchun Technology Correspondent Project(2009245).

** Research direction: Expert Systems, Data Mining, Precision Agriculture.
} 
information network construction. According to the current problems in construction of agricultural information website, this paper apply the storage structure of cloud computing server to digital agricultural information resources sharing, and analyze the Open Grid Services Architecture. On this basis, by using virtual machine way, we virtualize the cloud computing server, plan overall structure of grid and resource sharing model, and focus on the applications of virtualization technology in information sharing services. Through the existing server virtualization, only one or a few servers can be realized that requires multiple servers running the service, it significantly reduced the overall cost including hardware acquisition, maintenance investment, management investment and energy consumption. Virtualization technology allows hardware resources will be well allocated[3]. Virtualization technology automatically deploy the hardware resources for the service so that improving the effective of utilization. It also improve the stability and security, provide the service independently in the operating system and operating environment, and reduce management complexity. At the same time, the security and reliability are guaranteed. Even if a service fails, just for processing this virtual machine, and do not affect other services.

Based on the complexity of agricultural information, ever-changing environment and the status and features of cloud computing, with the applications of cloud computing, we proposed digital agricultural information resource sharing plan. It makes the further sharing of agricultural information resources and will play a more active and effective role for agricultural production decisions, agricultural extension, agricultural product sales and other information services.

\section{Basic Theory}

Cloud computing is first presented in 2006 Amazon's Elastic Compute Cloud, which uses virtual machine technology to provide low-cost network but better quality of service for users[4]. While cloud computing was not first proposed as efficient computational model, but it greatly promote the popularization of the Infrastructure-as-a-Service (IaaS).

\subsection{Concepts}

Cloud Computing is the fusion of Grid Computing, Distributed Computing, Parallel Computing, Utility Computing, Network Storage Technology, Virtualization, Load Balance and other traditional computer and network technology[5,6]. Narrow Cloud Computing is the delivery of IT infrastructure and usage patterns, refers to the network on-demand and scalable way to obtain the necessary resources (hardware, platform, software). Generalized Cloud computing refers to the broad service delivery and usage patterns, that is, by on-demand and scalable way, through the network to obtain the necessary services. Such services can be IT and software, related internet, you can also make any other services.

\subsection{Features}

The important idea of cloud computing is to connect a large number of computing resources with a unified network management and scheduling, to form a pool of 
computing resources on-demand services for users[7].The network providing resources is called "cloud". The resources in cloud are infinitely expanded in the user location, and can be obtained at any time, on-demand used, at any time extended and paid per use. This feature is often referred to as water and electricity as the use of IT infrastructure. Cloud computing break the traditional Internet sharing restrictions on the network and achieve a unified management of heterogeneous resources. According to the user's demand, the users do not perceive their own location and other details of the application, can obtain the corresponding services through the connected network.

\subsection{Composition}

Cloud computing model mainly consists of three parts: the client, the transmission network and the server [8]. The front-end user show is PC and other devices, which is known as a client and may not load the application. The middle transmission network includes a variety of wired and wireless network transmission facilities to implement the data transfer between the client and the server, while the users do not realize that the network exists. The server in cloud computing model is very important for cloud computing, operating system, application and services, the data users need are stored on the server, it can provide better service.

\section{Research on Digital Agricultural Information Resources Sharing Plan}

Combining the demand for agricultural information resource sharing with the features of cloud computing model, we designed a platform for digital agricultural information resources sharing plan, the overall platform framework are shown in Figure 1.

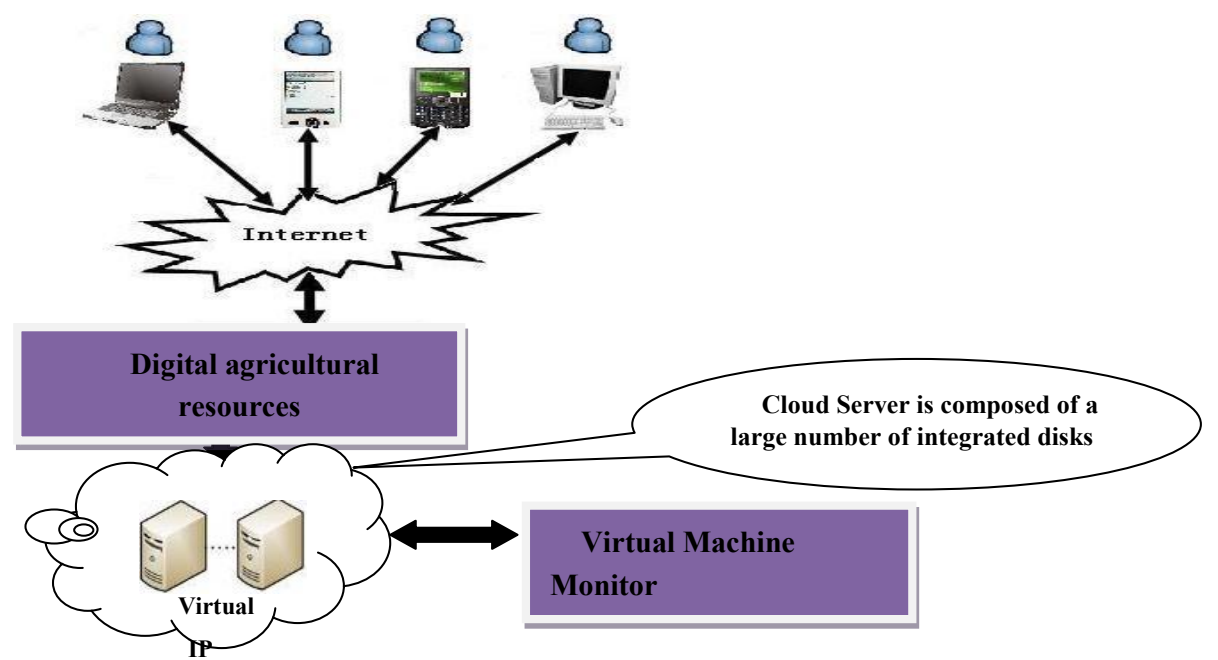

Fig. 1. The general framework for the platform of cloud computing 
The server is built using the integrated hard disks, pre-stored a variety of operating platforms including operating system environment, applications and the corresponding digital information. The open source software XEN[9] implement the server virtualization, and build storage cloud of digital agricultural information resources. The platform has a good dynamic, scalability and flexibility. The server can timely publish information resources, the users can choose their own interest information resources, so that each user obtain and build the operating environment from the server to meet the demand for different information resources, to achieve the purpose of heterogeneous platforms.

\subsection{Cloud Computing Server Storage Structure}

In the process of building a cloud computing servers, we can use the large servers. Applying this approach, although the storage capacity meet the requirements of the users, the expense is relatively too much. With the expansion of hard disks capacity and the falling of hard disks prices on the market, we can integrate a lot of hard disks together and build a large capacity and low-cost server.

As the requests between users are isolated from each other, so the server can select the independent user to store relatively, the whole system can be extended to large scale. On the server side, we can distribute the digital agricultural information to multiple hard disks of multiple nodes, and allow users to read and write in multiple hard disks. We can handle redundant error information on the server in order to ensure user to access the safe and effective information.

\subsection{Cloud Computing Server Virtualization}

Through open source software XEN, we can implement the server virtualization and build storage cloud of digital agricultural information resources, the virtual configuration shown in Figure 2.

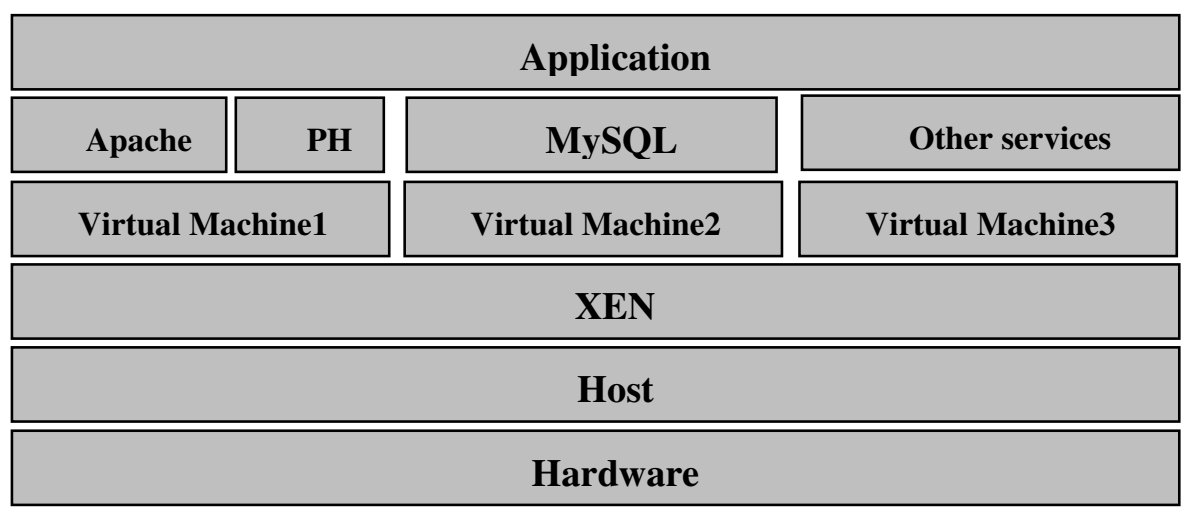

Fig. 2. The configuration diagram of virtualization 
This structure need to configure three virtual machines running different services. Their functions are:

Virtual Machine1 is responsible for Http service, running Apache and PHP.

Virtual Machine2 is responsible for database services, using MySQL.

Virtual machine3 is responsible for other services, such as email servers, etc.

PHP is a script interpreter which run in the manner of Apache module, so Apache and PHP will be deployed in the same virtual machine at the same time.

\subsection{Information Resource Sharing Plan}

On the basis of these researches, we take retrieval module of digital agricultural information resources for example, and design digital agricultural information resources sharing services plan. Through virtualization technology the users can manage interface to search agricultural information. Although the information services from cloud in different locations[10], the user do not need to know the specific location of the distribution of the information. The information is completely transparent for users, which feel as easy as accessing the database on the local computer and publish the retrieved results in time. The process of resources retrieval and publication is shown in Figure 3.

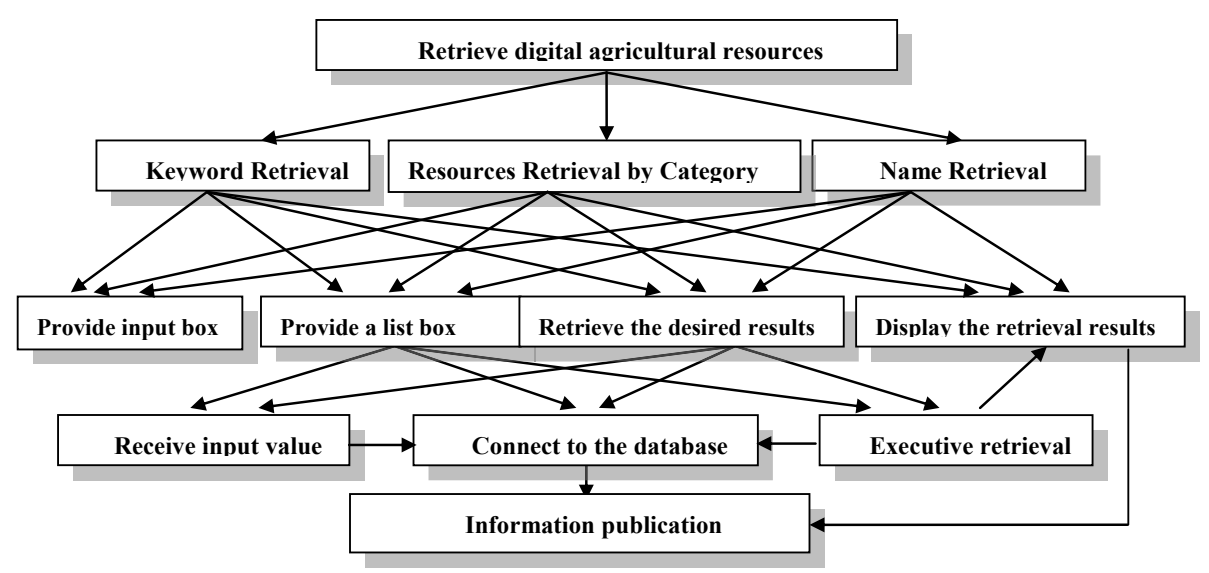

Fig. 3. The retrieval and publish of the resources

\section{$4 \quad$ Application Examples}

This paper apply digital agricultural information resources sharing plan based on cloud computing in Jilin digital agricultural information website construction. Jilin digital agricultural information website mainly consist of digital agricultural information vertical portal module, CA certificate management module, digital agricultural 
resources retrieval module, digital agricultural data analysis module, expert answers service module, personalized service module and agricultural BBS forum module.

The site uses the Linux operating system, cloud computing, virtualization technology and Mysql, Arcgis server database. It realizes communication of digital agricultural information resources and provides users with a unified and transparent services to achieve maximum resource sharing. System's main interface shown in Figure 4.

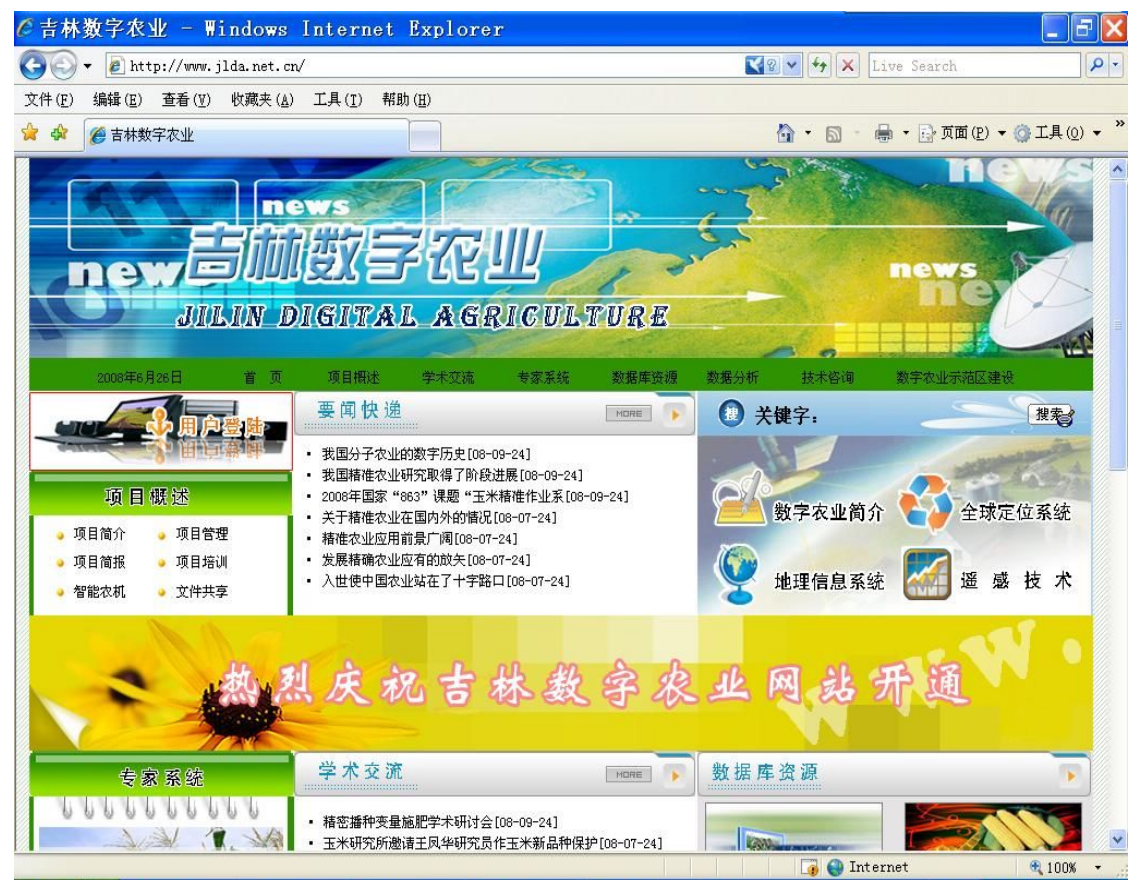

Fig. 4. The home page of website

Taking agricultural resources retrieval module and data analysis module for example, we illustrate the main functions of this site.

\subsection{Agricultural Resources Retrieval Module}

This module provides retrieval services of the resources, through the use of keyword retrieval, resource retrieval by category and name retrieval, the users can obtain the corresponding information. After the users submit a search condition, the system will implement retrieval process based on submitted information, and output the required information in a timely manner. The retrieval interface shown in Figure 5. 


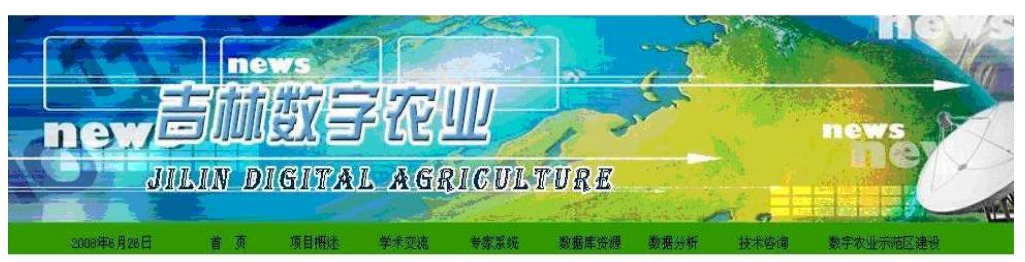

\section{农业资源检索}

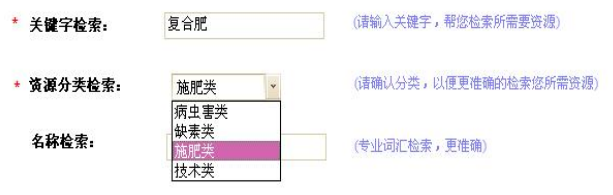

Fig. 5. The interface of information retrieval

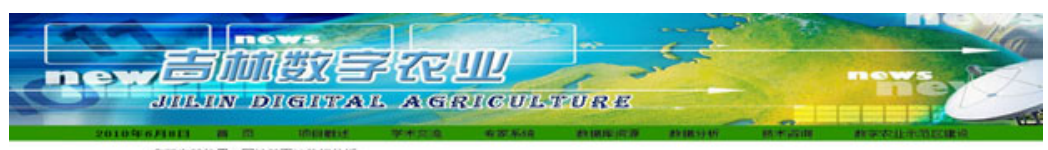

suxame

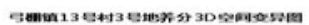

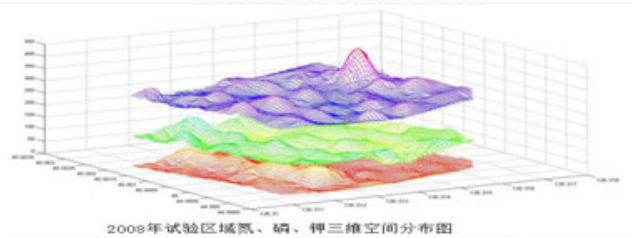

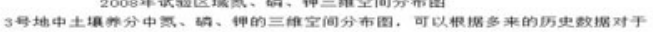

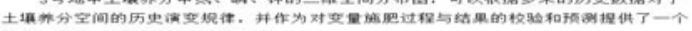

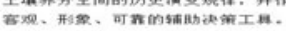

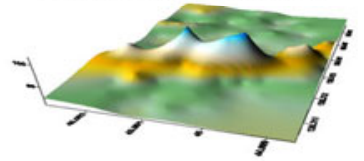

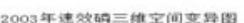

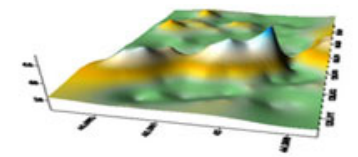

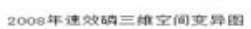

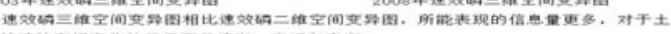
㙁逢

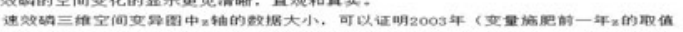

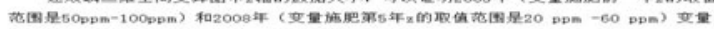

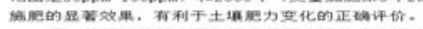

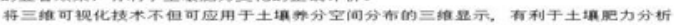

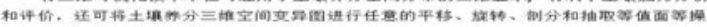

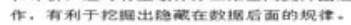

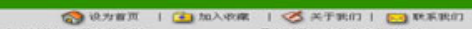

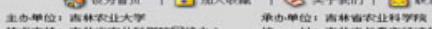

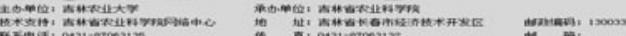

Fig. 6. The interface of data analysis 


\subsection{Data Analysis Module}

This module can analyze the soil historical nutrient data such as nitrogen, phosphorus, potassium, organic matter, moisture and ph value, and generate two-dimensional and three-dimensional nutrient maps for many years. These maps can clearly, intuitively, really represent soil nutrients historical evolution, and provide objective and reliable decision support for the validation and prediction of variable rate fertilization process and results. Data analysis interface shown in Figure 6.

\section{Conclusion}

Through research on cloud computing and virtualization services, this paper present digital agricultural information resources sharing plan based on cloud computing. For the emerging field of digital agriculture, we establish a professional information resource sharing platform among agricultural enterprises, units, research staff and users. We also build a powerful polymeric platform of information resources retrieval, communication and sharing. The platform has a good dynamic, scalability and flexibility, the server can timely publish information resources, and the users can choose their own interest information resources. This paper has done the following work:

1.Using the integrated hard disks to build cloud computing servers, pre-stored a variety of different operating platforms, including operating system environment, applications and the corresponding digital agricultural information.

2.Utilizing a virtual machine, the open source software XEN implement the server virtualization, we build the efficient and low- cost virtual structure and storage cloud of digital agricultural information resources.

3.With the storage structure of cloud computing and virtual deployment, we design a cost-effective, high efficiency and easy to manage solution, achieve the sharing of agricultural information resources, and provide a personalized, visual, multi-prospective and multi-level active service model.

Our agriculture is focusing on the construction of agricultural information website. As the rapid development of agricultural sites in our country today, focusing on digital agricultural information resources sharing plan, establishing the authority of the agricultural information and providing services for agricultural employees, will be bound to do its due contribution for our agricultural development.

\section{References}

1. He, Y.-J., Zhang, J.-H.: Research on Operation Situation Development Strategy of Chinese Agricultural Information Website. Journal of Huazhong Agricultural University (Social Science Edition) 3(49), 12-14 (2003)

2. Feng, D.-G., Zhang, M., Zhang, Y., Xu, Z.: Study on Cloud Computing Security. Journal of Software 22(1), 71-83 (2011) 
3. Rosenthal, A., Mork, P., Li, M.H., Stanford, J., Koester, D., Reynolds, P.: Cloud computing: A new business paradigm for biomedical information sharing. Journal of Biomedical Informatics (43), 342-353 (2010)

4. Mateescua, G., Gentzsch, W., Ribbens, C.J.: Hybrid Computing-Where HPC meets grid and Cloud Computing. Future Generation Computer Systems 27, 440-453 (2011)

5. Zissis, D., Lekkas, D.: Addressing cloud computing security issues. Future Generation Computer Systems, 1-10 (2010)

6. Murphy, M.A., Goasguen, S.: Virtual Organization Clusters: Self-provisioned clouds on the grid. Future Generation Computer Systems (26), 1271-1281 (2010)

7. Vázquez, C., Huedo, E., Montero, R.S., Llorente, I.M.: On the use of clouds for grid resource provisioning. Future Generation Computer Systems (27), 600-605 (2011)

8. Chen, K., Zheng, W.-M.: Cloud Computing: System Instances and Current Research. Journal of Software 20(5), 1337-1348 (2009)

9. Li, Y.-Q., Song, Y., Huang, Y.-B.: A Memory Global Optimization Approach in Virtualized Cloud Computing Environments. Chinese Journal of Computers 34(4), 684-693 (2011)

10. Tian, G.-H., Meng, D., Zhan, J.-F.: Reliable Resource Provision Policy for Cloud Computing. Chinese Journal of Computers 33(10), 1859-1872 (2010) 